\title{
Visualizing Biochemical Activities in Living Cells through Chemistry
}

\author{
Luc Reymond, Gražvydas Lukinavičius, Keitaro Umezawa, Damien Maurel, Matthias A. Brun, \\ Anastasiya Masharina, Karolina Bojkowska, Birgit Mollwitz, Alberto Schena, Rudolf Griss, and \\ Kai Johnsson*
}

\begin{abstract}
The development of molecular probes to visualize cellular processes is an important challenge in chemical biology. One possibility to create such cellular indicators is based on the selective labeling of proteins with synthetic probes in living cells. Over the last years, our laboratory has developed different labeling approaches for monitoring protein activity and for localizing synthetic probes inside living cells. In this article, we review two of these labeling approaches, the SNAP-tag and CLIP-tag technologies, and their use for studying cellular processes.
\end{abstract}

Keywords: Cell biology $\cdot$ Imaging $\cdot$ Protein chemistry $\cdot$ SNAP-tag $\cdot$ Synthetic probes

\section{Introduction}

Autofluorescent proteins (AFPs) such as green fluorescent protein (GFP) are only mediocre fluorophores compared to synthetic fluorescent dyes. Yet they have become the fluorophores of choice for most applications in biology. ${ }^{[1]}$ The reason for this is the possibility to genetically encode AFPs and thereby through simple genetic engineering fuse them to other proteins in living cells. This enables an almost unlimited number of applications, ranging from the specific fluorescent labeling of proteins of interest to the generation of sophisticated fluorescent sensors for biochemical activities. Inspired by the success of AFPs, chemists developed approaches that combine the advantages of genetic engineering with the potential offered by synthetic probes. ${ }^{[2]}$ These hybrid approaches are based on the specific reaction of a polypeptide with a synthetic substrate; derivatizing such a substrate with a synthetic probe and expressing the polypeptide (often referred to as a tag) in fusion with a protein of interest results in a specific targeting of the probe to the protein of interest. To be of practical utility for applications in living cells, such an approach must fulfill a number of criteria: First, the rate of reaction between the tag and its substrate must be sufficiently fast at low micromolar concentrations of both reaction partners so that quantitative labeling can be achieved within minutes. Second, the reaction must be of sufficient selectivity so that in a cellular environment only the tag interacts with the substrate. At the same time, the derivatization of the substrate with different synthetic probes should be possible without significantly affecting speed and selectivity of the reaction. Furthermore, the substrate should possess good cell permeability and minimal toxicity. The first labeling approach that fulfilled at least partially these crite- ria was introduced by the group of Roger Tsien in 1998. ${ }^{[3]}$ The approach is based on the selective chelation of biarsenical fluorophores by a polypeptide containing four appropriately spaced cysteine residues, the so-called tetracysteine tag. In 2003, our group introduced SNAP-tag, an engineered $\mathrm{O}^{6}$-alkylguanine-DNA alkyltransferase (AGT) that specifically reacts with $\mathrm{O}^{6}$-benzylguanine (BG) derivatives (Fig. 1). ${ }^{[4]}$ Wild-type AGT repairs mutagenic and cytotoxic DNA lesions that result from $\mathrm{O}^{6}$ alkylation of guanine. Repair is achieved by transferring the alkyl group to a reactive cysteine; the alkylated AGT is subsequently not regenerated. Taking advantage of this unusual DNA repair mechanism, we generated SNAP-tag by engineering wild- (a)

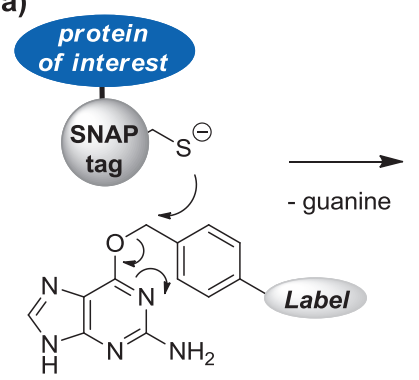

(b)

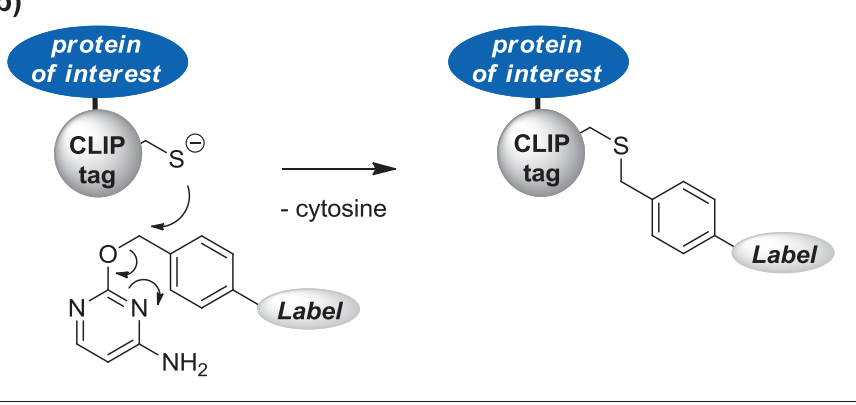

Fig. 1. Labeling mechanism of SNAPtag (a) and CLIP-tag (b) fusion proteins. 
type AGT for an efficient reaction with BG derivatives carrying synthetic probes, leading to a covalently labeled tag. SNAPtag is a monomeric protein of 182 residues $(20 \mathrm{kDa})^{[5]}$ that can be efficiently labeled in living cells and even in vivo. SNAP-tag has become a widely accepted tool in life sciences, with recent applications ranging from measurement of protein half-lives in animals to superresolution microscopy and single molecule spectroscopy.[6]

To enable the specific and simultaneous labeling of two different proteins of interest with different probes, our group introduced in 2008 the CLIP-tag. ${ }^{[7]}$ CLIPtag was generated by engineering the substrate specificity of SNAP-tag so that it specifically reacts with $\mathrm{O}^{2}$-benzlycytosine (BC) derivatives. SNAP-tag and CLIP-tag fusion proteins can be specifically and simultaneously labeled with BG and BC derivatives in cells. One of the applications of such a double labeling is the characterization of protein-protein interactions through Förster resonance energy transfer (FRET). ${ }^{[8]}$

Acceptance of chemistry-based technologies in the life science community depends on the commercial availability of the necessary reagents. Fortunately, a large number of different substrates for labeling of SNAP-tag and CLIP-tag fusion proteins are distributed by New England Biolabs, enabling biologists with no expertise in synthetic chemistry to apply these technologies to their research. By discussing recent work from our laboratory, we will highlight how SNAP-tag and CLIP-tag can be exploited to study a wide variety of different problems in biology.

\section{Localizable Calcium Indicators}

Critical for the role of calcium $\left(\mathrm{Ca}^{2+}\right)$ as a second messenger is a precise spatial and temporal control of its concentration in cells. ${ }^{[9]}$ Local $\mathrm{Ca}^{2+}$ concentrations $\left(\left[\mathrm{Ca}^{2+}\right]\right.$ ) in the cell can rise within milliseconds by orders of magnitudes. There are two main classes of fluorescent probes to measure fluctuations in $\left[\mathrm{Ca}^{2+}\right]$ : synthetic $\mathrm{Ca}^{2+}$ indicators such as Fura-2 and Fluo-4 or AFPbased $\mathrm{Ca}^{2+}$ indicators such as cameleon and GCamP2.[10] AFP-based sensors can be genetically targeted to an area of interest in cells, but have slower response time and lower sensitivity than synthetic indicators. In contrast, synthetic indicators lack the ability to be specifically localized in cells. The targeting of synthetic $\mathrm{Ca}^{2+}$ indicators through coupling to localized protein tags potentially combines the advantages from both synthetic and AFP-based indicators. ${ }^{[11]}$ We have recently developed ratiometric and intensity-based fluorescent $\mathrm{Ca}^{2+}$ indicators that can be covalently coupled to

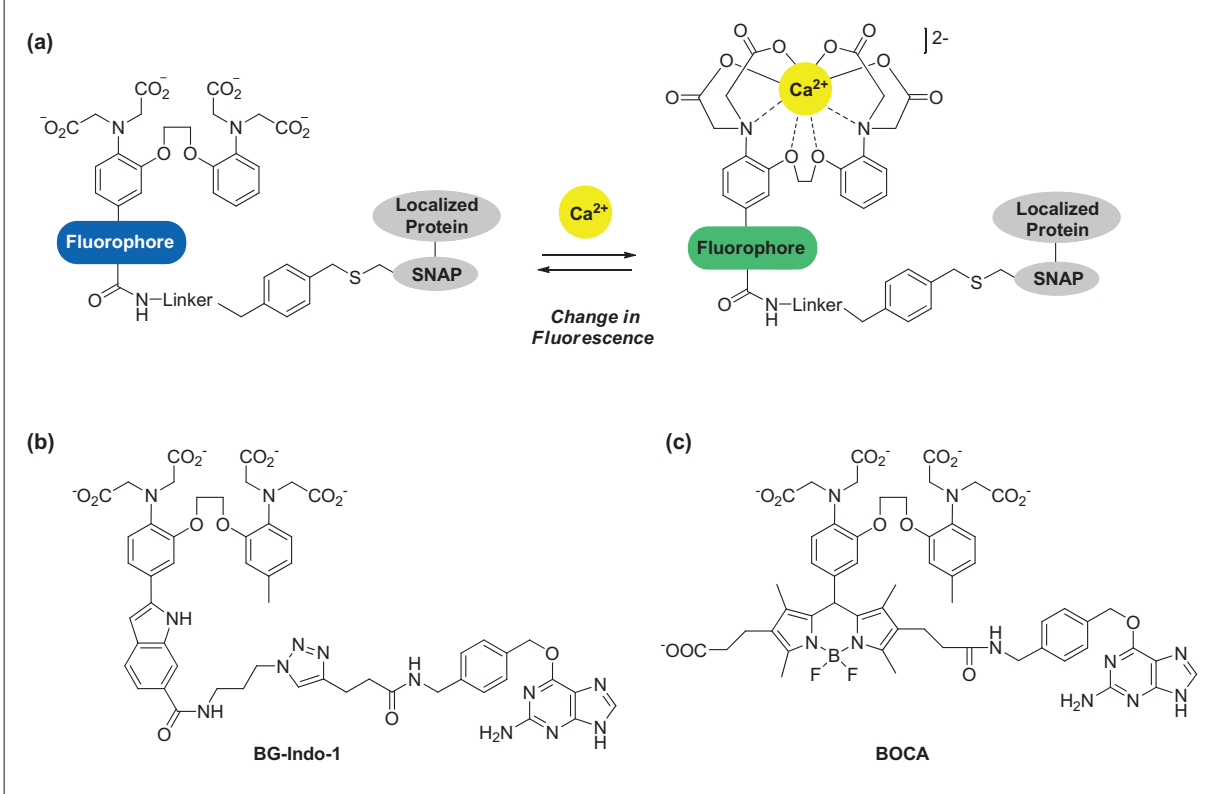

Fig. 2. (a) Localizable calcium indicators. (b) Structure of BG-Indo-1. (c) Structure of BODIPYbased calcium indicator (BOCA).

SNAP-tag fusion proteins in living cells (Fig. 2a). ${ }^{[12]}$ Indo-1 is a useful synthetic $\mathrm{Ca}^{2+}$ indicator whose emission spectrum depends on $\left[\mathrm{Ca}^{2+}\right]$ and that therefore can be used for ratiometric measurements. ${ }^{[13]}$ To localize Indo-1 in living cells we have synthesized a BG-Indo-1 derivative that can be coupled to SNAP-tag fusion proteins and retains its $\mathrm{Ca}^{2+}$-sensing ability (Fig 2b). ${ }^{[12 \mathrm{a}]}$ Local $\mathrm{Ca}^{2+}$ sensing was demonstrated in cultured primary muscle cells of mice expressing a nucleus-localized SNAP-tag fusion. While ratiometric $\mathrm{Ca}^{2+}$ indicators are useful for determining absolute $\left[\mathrm{Ca}^{2+}\right]$, they are less sensitive than intensity-based synthetic fluorescent indicators. We therefore introduced a synthetic $\mathrm{Ca}^{2+}$ indicator based on a BODIPY fluorophore that shows a large, 180-fold fluorescence increase upon $\mathrm{Ca}^{2+}$ binding when coupled to SNAP-tag fusion proteins (Fig. 2c). ${ }^{[12 b]} \mathrm{We}$ further demonstrated how the SNAP-tagbound sensor can be used to sense changes in $\left[\mathrm{Ca}^{2+}\right]$ in the nuclei and in the cytosol of live CHO-K1 cells. Our hybrid indicators combine the spatial specificity of biosensors with the fast kinetics and high dynamic range of small synthetic indicators. These features should make them a valuable addition to the existing methods to sense $\left[\mathrm{Ca}^{2+}\right]$ in living cells.

\section{Photoactivatable Probes for Protein Labeling}

Photosensitive fluorescent proteins (pFPs) are autofluorescent proteins whose fluorescent properties can be activated or switched with light.[14] Examples include photoactivatable GFP, which can be trans- formed with UV light from a dark to a highly fluorescent state, and variants of Eos, whose emission and excitation wavelength can be red-shifted with a light pulse. pFPs have become important tools in biological research as they permit to highlight subpopulations of a given protein with optical methods in living cells. An alternative approach to generate photosensitive proteins is the specific labeling of proteins with photosensitive synthetic fluorophores. We have recently introduced a generally applicable strategy for the generation of photoactivatable and photoconvertible fluorescent probes that can be selectively coupled to SNAP-tag fusion proteins in living cells (Fig. 3a). ${ }^{[15]}$ The photosensitivity of the probes is based on photocleavage of a linker between a quencher or a second fluorophore (the acceptor) and the fluorophore of interest (the donor). Prior to photocleavage, FRET from the donor to the acceptor quenches the fluorescence of the donor; after photocleavage the donor becomes highly fluorescent and remains attached to SNAPtag (Fig. 3a). Following this design principle, photoactivatable versions of fluorescein and $\mathrm{Cy} 3$ as well as a photoconvertible Cy5Cy3 probe were synthesized and coupled to selected proteins on the cell surface, in the cytosol and in the nucleus of cells. We employed the photoactivatable $\mathrm{Cy} 3$ probe to measure the mobility of cell surface receptors. The approach can be extended to a large variety of fluorophores and thereby establishes a generally applicable strategy for the generation of photosensitive and localizable fluorophores with tailor-made properties.

The imaging of biological structures with a resolution below the diffraction limit, i.e. superresolution microscopy, is probably the most important development 
in the field of fluorescent microscopy in the last years.[16] Stochastic Optical Reconstruction Microscopy (STORM) is one of the different approaches for superresolution microscopy. ${ }^{[17]}$ STORM requires the use of reversibly photoswitchable fluorophores so that only an optically resolvable subset of fluorophores is activated at any moment of time. Superresolution imaging is achieved by precise mathematical localization of single emitters across all acquired frames. In STORM, the selective attachment of suitable fluorophores is one of the main challenges of the approach. We have introduced photoswitchable probes for the labeling of SNAP-tag fusion proteins and successfully used them for STORM-based superresolution microscopy (Fig. 3b). ${ }^{[18]}$ Using these probes, we imaged microtubules in fixed cells with a resolution of about $40 \mathrm{~nm}$. In general, the superior brightness of synthetic fluorophores over AFPs makes them particularly well suited for superresolution microscopy which should therefore become an important field of use for protein labeling as it offers a straightforward solution to localize such fluorophores.

\section{Identifying Protein-Protein Interactions through Specific Crosslinking}

Protein-protein interactions play important roles in almost all biological processes. The identification and characterization of protein-protein interactions is therefore an important challenge in molecular biology. As existing approaches often fail to detect physiologically relevant interactions, there is a generally acknowledged need for new and complementary approaches to detect protein-protein interactions. We recently introduced a technically simple method that enables the sensitive detection of protein-protein interactions in cell lysates through the selective crosslinking (S-CROSS) of protein complexes. ${ }^{[19]}$ $\mathrm{S}-\mathrm{CROSS}$ is based on the co-expression of proteins fused to SNAP-tag or CLIP-tag and the lysis of cells in the presence of a crosslinker in which the substrates of the two tags are connected via a fluorophore (Fig. 4). The efficiency of the resulting crosslinking of fusion proteins depends on their proximity and can be quantified after SDS gel electrophoresis by in-gel fluorescence scanning. Crosslinking efficiency can be used as an indicator of interaction between two proteins, thereby allowing the identification of interacting protein pairs. We validated S-CROSS by confirming various interactions through selective crosslinking and showed that it permits the quantitative and simultaneous analysis of multiple protein complexes. An ad-

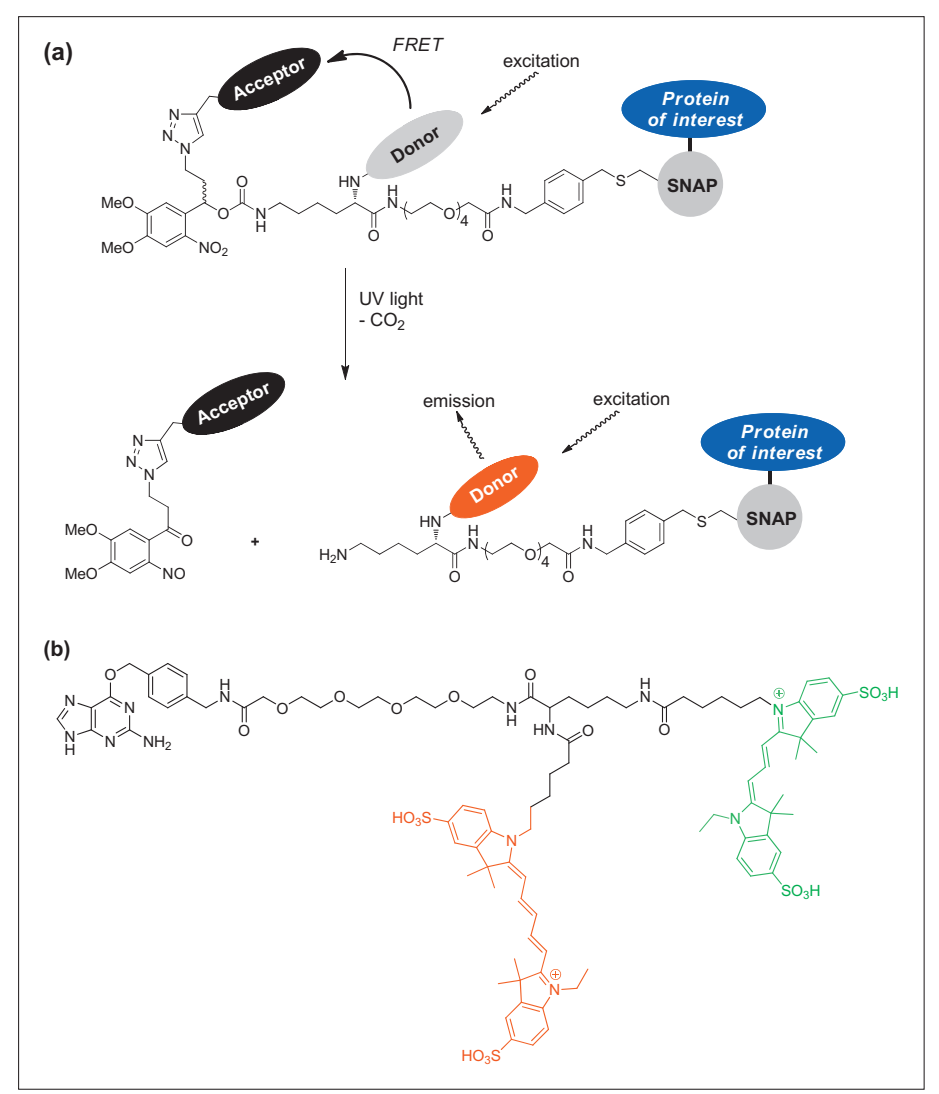

Fig. 3. (a)

Photoswitchable probes for protein labeling. (b) SNAPtag substrate for Stochastic Optical Reconstruction Microscopy (STORM).

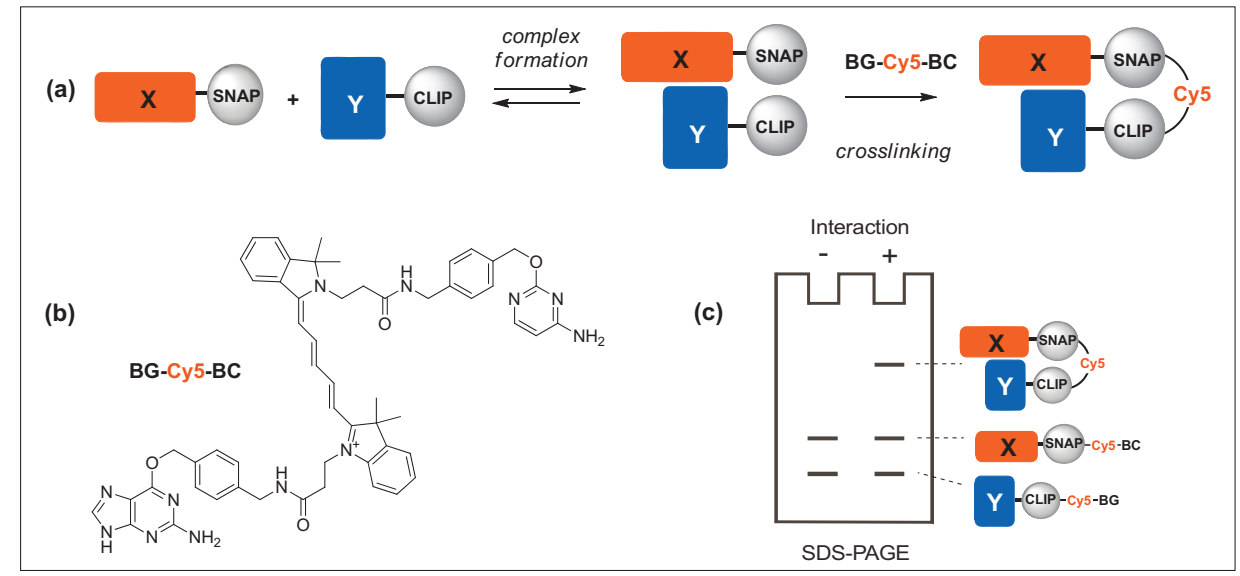

Fig. 4. (a) Selective crosslinking of SNAP-tag and CLIP-tag fusion proteins for the detection of protein-protein interactions. (b) Structure of crosslinker. (c) Illustration of how selective crosslinking is analyzed by gel electrophoresis and subsequent in-gel fluorescence scanning.

ditional attractive feature of the approach is the possibility to exploit the versatility of chemical labeling to complement crosslinking studies with live cell imaging to determine, for example the co-localization of protein pairs.

\section{Snifits: New Fluorescent Sensors for Metabolites}

An animal cell contains thousands of metabolites whose concentrations fluctuate over time. Tracking the concentrations of metabolites with spatiotemporal resolution in living cells and in vivo is an important challenge in biology. AFP-based sensor proteins offer the possibility to probe the concentration of key metabolites in living cells. ${ }^{[20]}$ These sensors are usually based on a conformational change of a protein upon metabolite binding. Sandwiching such a binding protein between two AFPs permits the detection of the metabolite of interest through changes in FRET efficiency. The obligatory conformational change in the binding protein severely limits the choice of proteins available for the development of new FRET biosensors. We took advantage of the opportunities offered by protein labeling to develop an approach that overcomes this limitation (Fig. 5). [21] Our Snifit biosensors (SNAP tag-based indicator proteins with a fluorescent intramolec- 


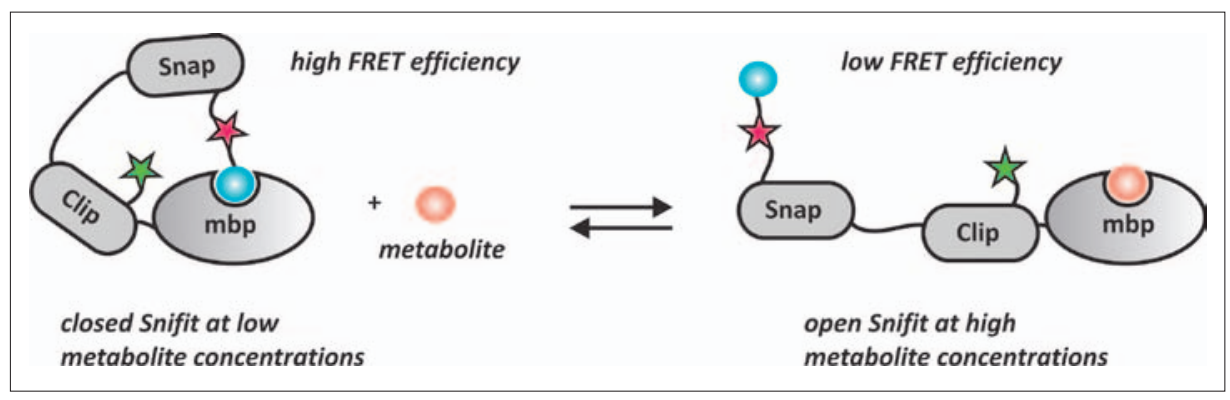

Fig. 5. Snifits, semisynthetic fluorescent sensor proteins. SNAP-tag is labeled with a molecule containing a fluorophore (red star) and a ligand (pink ball) that can bind to the metabolite-binding protein (mbp). CLIP-tag is labeled with a second fluorophore (green star). The Snifit can be transformed from a closed to an open conformation through binding of free metabolite (blue ball). Metabolite concentrations are determined by measuring FRET efficiency of the sensor.

ular tether) are comprised of i) SNAP-tag, ii) CLIP-tag, iii) a binding protein, and iv) a synthetic molecule containing a fluorophore and a ligand that can bind to the binding protein (Fig. 5). The synthetic molecule is coupled to SNAP-tag whereupon the ligand can bind intramolecularly to the binding protein. CLIP-tag is labeled with a fluorophore that forms a good FRET pair with the fluorophore attached to SNAPtag. In the absence of analyte, the Snifit exists in a closed conformation whereas in the presence of analyte the equilibrium is shifted towards an open conformation. The opening and closing of the Snifit leads to a change in the position of the two fluorophores relative to each other and in the FRET efficiency between them. We have demonstrated that Snifits for different key metabolites, such as neurotransmitters, can be generated and that these sensors can be assembled in living cells and on cell surfaces. We foresee that Snifits will become important tools for probing previously inaccessible metabolites.

\section{Identifying Drug-Protein Interactions with a SNAP-based Yeast Three-hybrid System}

The identification of the protein targets of a given drug provides the basis for the understanding of its mechanism of action. However, the identification of drug-protein interactions remains a challenging task as testified by the fact that the primary targets of various clinically approved drugs remain unknown. We have recently introduced a SNAP-tag-based yeast three-hybrid ( $\mathrm{Y} 3 \mathrm{H})$ system for identification of drug-protein interactions. ${ }^{[22]}$ The $\mathrm{Y} 3 \mathrm{H}$ system is a modified version of the yeast two-hybrid (Y2H) system adapted for detection of drug-protein interactions. In our $\mathrm{Y} 3 \mathrm{H}$ system the drug of interest is derivatized with BG and anchored to a DNA-binding protein inside yeast cells via SNAP-tag. ${ }^{[22,23]}$ The interaction of the anchored drug with a target protein is then detected by linking their association to the transcriptional activation of a reporter gene. We have demonstrated the utility of this system by screening a number of clinically approved drugs for binding proteins, thereby identifying a number of previously unknown but physiological relevant drug-protein interactions. The experimental simplicity of the system and the possibility to use the same BG drug derivatives also for traditional affinity purifications of drug targets adds to the appeal of the approach.

\section{Conclusions}

The specific labeling of proteins with synthetic probes is a powerful tool to visualize biochemical activities in living cells. A number of complementary labeling technologies have been established over the last years and future research in this field should focus on the nature of the probes attached to the protein of interest. We hope that more chemists will take advantage of this exciting opportunity to design innovative synthetic probes for resolving important biological questions.

\section{Acknowledgment}

The authors acknowledge support from the Swiss National Science Foundation and EPFL.

Received: August 28, 2011 Tsien, Nat. Rev. Mol. Cell. Biol. 2002, 3, 906.

[2] M. J. Hinner, K. Johnsson, Curr. Opin. Biotechnol. 2010, 21, 766 .

[3] B. A. Griffin, S. R. Adams, R. Y. Tsien, Science 1998, 281, 269.

[4] A. Keppler, S. Gendreizig, T. Gronemeyer, H. Pick, H. Vogel, K. Johnsson, Nat. Biotechnol. 2003, 21,86 .

[5] A. Juillerat, C. Heinis, I. Sielaff, J. Barnikow, H. Jaccard, B. Kunz, A. Terskikh, K. Johnsson, Chembiochem 2005, 6, 1263.

[6] a) K. Bojkowska, F. Santoni de Sio, I. Barde, S. Offner, S. Verp, C. Heinis, K. Johnsson, D. Trono, Chem. Biol. 2011, 18, 805; b) A. A. Hoskins, L. J. Friedman, S. S. Gallagher, D. J. Crawford, E. G. Anderson, R. Wombacher, N.
[1] J. Zhang, R. E. Campbell, A. Y. Ting, R. Y.
Ramirez, V. W. Cornish, J. Gelles, M. J. Moore, Science 2011, 331, 1289; c) S. A. Jones, S. H. Shim, J. He, X. Zhuang, Nat. Methods 2011, 8 , 499.

[7] A. Gautier, A. Juillerat, C. Heinis, I. R. Correa, Jr., M. Kindermann, F. Beaufils, K. Johnsson, Chem. Biol. 2008, 15, 128.

[8] E. Doumazane, P. Scholler, J. M. Zwier, T. Eric, P. Rondard, J. P. Pin, FASEB J. 2011, 25, 66.

[9] D. E. Clapham, Cell 2007, 131, 1047.

[10] M. Mank, O. Griesbeck, Chem. Rev. 2008, 108 , 1550.

[11] O. Tour, S. R. Adams, R. A. Kerr, R. M. Meijer, T. J. Sejnowski, R. W. Tsien, R. Y. Tsien, Nat. Chem. Biol. 2007, 3, 423

[12] a) M. Bannwarth, I. R. Correa Jr., M. Sztretye, S. Pouvreau, C. Fellay, A. Aebischer, L. Royer, E. Rois, K. Johnsson, ACS Chem. Biol. 2009, 4 , 179; b) M. Kamiya, K. Johnsson, Anal. Chem. 2010, 82, 6472; c) A. A. Ruggiu, M. Bannwarth, K. Johnsson, Org. Biomol. Chem. 2010, 8 , 3398.

[13] G. Grynkiewicz, M. Poenie, R. Y. Tsien, J. Biol. Chem. 1985, 260, 3440.

[14] N. C. Shaner, G. H. Patterson, M. W. Davidson, J. Cell Sci. 2007, 120, 4247.

[15] D. Maurel, S. Banala, T. Laroche, K. Johnsson, ACS Chem. Biol. 2010, 5, 507.

[16] S. W. Hell, Science 2007, 316, 1153.

[17] M. Bates, B. Huang, G. T. Dempsey, X. Zhuang, Science 2007, 317, 1749

[18] C. Dellagiacoma, G. Lukinavicius, N. Bocchio, S. Banala, S. Geissbuhler, I. Marki, K. Johnsson, T. Lasser, Chembiochem 2010, 11, 1361.

[19] A. Gautier, E. Nakata, G. Lukinavicius, K. T. Tan, K. Johnsson, J. Am. Chem. Soc. 2009, 131, 17954.

[20] M. Fehr, D. W. Ehrhardt, S. Lalonde, W. B. Frommer, Curr. Opin. Plant Biol. 2004, 7, 345.

[21] M. A. Brun, K. T. Tan, E. Nakata, M. J. Hinner, K. Johnsson, J. Am. Chem. Soc. 2009, 131, 5873.

[22] C. Chidley, H. Haruki, M. G. Pedersen, E. Muller, K. Johnsson, Nat. Chem. Biol. 2011, 7, 375.

[23] S. Gendreizig, M. Kindermann, K. Johnsson, $J$. Am. Chem. Soc. 2003, 125, 14970. 